Research Article

\title{
ASC Performance Prediction for Medical IoT Communication Networks
}

\author{
Fagen Yin $\mathbb{D},{ }^{1}$ Pingping Xiao $\mathbb{D}^{\mathbb{D}},{ }^{1}$ and Zefeng $\mathrm{Li} \mathbb{C}^{2}$ \\ ${ }^{1}$ College of Physical Science and Engineering, Yichun University, Yichun 336000, Jiangxi, China \\ ${ }^{2}$ Institute of Data Science, City University of Macau, Macau 999078, Macau \\ Correspondence should be addressed to Fagen Yin; ynx522@163.com
}

Received 13 April 2021; Accepted 21 May 2021; Published 28 May 2021

Academic Editor: Xingwang Li

Copyright (c) 2021 Fagen Yin et al. This is an open access article distributed under the Creative Commons Attribution License, which permits unrestricted use, distribution, and reproduction in any medium, provided the original work is properly cited.

Wearable devices are gradually entering the medical health field. Medical Internet of Things (IoT) has been widely used in all walks of medical health. With the complexity of medical health application scenarios, the medical IoT communication networks face complex environments. The secure communication issue is very important for medical IoT communication networks. This paper investigates the secrecy performance of medical IoT communication networks. To improve the secrecy performance, we adopt a cooperative communication strategy. We also use the average secrecy capacity (ASC) as a metric, and the expressions are first derived. Then, a secrecy performance intelligent prediction algorithm is proposed. The extensive simulations are used to verify the proposed method. Compared with other methods, the proposed algorithm realizes a better prediction precision.

\section{Introduction}

With explosive growth of medical health applications, the fifth-generation (5G) mobile communication has been widely used in medical Internet of Things (IoT) networks $[1,2]$. Different 5G applications [3-5] widely appear in medical IoT communication networks, which can provide quick and convenient user experience and services [6]. However, due to the medical user mobility, the secure communication issue of medical IoT networks is facing many challenges [7].

For medical IoT communication networks, physical layer security is becoming more and more important [8]. With an eavesdropper, the authors [9] investigated the impact of antenna correlation. In [10], the authors developed a code scrambling scheme and analyzed secrecy performance. Considering the physical layer security, Yan et al. [11] studied the resource allocation problem for the cognitive relay networks. In [12], the authors proposed an optimal power allocation to achieve the secure transmission. Considering the cooperative jamming, $\mathrm{Lu}$ et al. [13] proposed a secure transmission scheme.
However, analyzing and predicting mobile secrecy performance are very difficult. Recently, machine learning techniques are applied in 5G wireless communications $[14,15]$. In pattern classification, classifying the binary data was realized by the support vector machine (SVM) model in [16]. The extreme learning machine (ELM) model was proposed to detect anomaly states in [17]. In [18], the general regression (GR) model predicted the video transmission quality.

To date, no existing studies have considered the secrecy performance prediction of AF relaying medical IoT communication networks. As a consequence, we summarize the main contributions as follows:

(1) The secrecy performance is analyzed with AF relaying scheme. Then, we use the average secrecy capacity (ASC) to evaluate secrecy performance and derive the exact expressions.

(2) To realize real-time analysis of ASC, we propose an ASC prediction algorithm based on the BP network. ELM, SVM, and GR methods are examined and compared with the proposed method. 
(3) We verify the derived ASC results under different conditions. Compared with different methods, the proposed algorithm realizes a better prediction precision and a lower time complexity.

\section{The Medical IoT Communication Network Model}

In Figure 1, the medical IoT communication networks have a mobile source (MS), mobile eavesdropper (ME), mobile destination (MD), and mobile relay (MR). $W_{\mathrm{SR}}, W_{\mathrm{RD}}$, and $W_{\mathrm{RE}}$ are the relative geometrical gains of $\mathrm{MS} \longrightarrow \mathrm{MR}, \mathrm{MR}$ $\longrightarrow \mathrm{MD}$, and $\mathrm{MR} \longrightarrow \mathrm{ME}$ links, respectively.

Transmission power is $E$, which is allocated by $K$. 2Rayleigh distribution can express the channel coefficient $h$ [19].

Firstly, MR receives the signal $r_{\mathrm{SR}}$ as [20]

$$
r_{S R}=\sqrt{W_{S R} K E} h_{S R} x+n_{S R},
$$

where $n_{\mathrm{SR}}$ is Gaussian noise.

In the second time slot, $\mathrm{AF}$ is used at MR. MD and ME receive the signals $r_{\mathrm{R} k i}, k \in\{\mathrm{D}, \mathrm{E}\}$, as

$$
r_{R k}=\sqrt{c_{k} E} h_{S R} h_{R k} x+n_{R k} .
$$

The received $\mathrm{SNR}_{\gamma \mathrm{SR} k i}$ is given as

$$
\gamma_{S R k}=\frac{\gamma_{S R \gamma R k}}{1+\overline{\gamma_{S R}}+\gamma_{R k}}
$$

where

$$
\begin{aligned}
& \gamma_{S R}=W_{S R} K\left|h_{S R}\right|^{2} \bar{\gamma}, \\
& \gamma_{R k}=(1-K) W_{R k}\left|h_{R k}\right|^{2} \bar{\gamma}, \\
& \overline{\gamma_{S R}}=W_{S R} K \bar{\gamma} .
\end{aligned}
$$

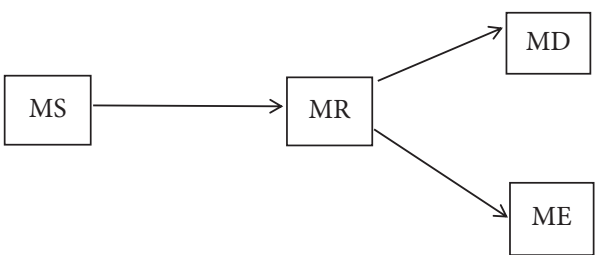

Figure 1: System model.

[21]

$\gamma_{\text {SRk }}$ is hard to calculate exactly. We approximate $\gamma_{\mathrm{SR} k i}$ as

$$
\begin{aligned}
\gamma_{S R A k} & =\frac{\gamma_{S R \gamma R k}}{1+\overline{\gamma_{S R}}+\overline{\gamma_{R k}}}, \\
\overline{\gamma_{R k}} & =(1-K) W_{R k} \bar{\gamma} .
\end{aligned}
$$

With the help of [22], the PDF and CDF of $\gamma_{\mathrm{SRA} k}$ are as follows:

$$
\begin{aligned}
& f_{\gamma_{S R A k}}(r)=\frac{1}{r} G_{0,4}^{4,0}\left[\frac{r}{\chi_{k}} \mid 1,1,1,1_{-}\right], \\
& F_{\gamma_{S R A k}}(r)=G_{1,5}^{4,1}\left[\frac{r}{\chi_{k}} \mid 1,1,1,1,0_{1}\right],
\end{aligned}
$$

where

$$
\chi_{k}=\frac{\overline{\gamma_{S R} \gamma_{R k}}}{1+\overline{\gamma_{S R}}+\overline{\gamma_{R k}}} .
$$

The instantaneous secrecy capacity is given as [23]

$$
C=\max \left\{\ln \left(1+\gamma_{S R A D}\right)-\ln \left(1+\gamma_{S R A E}\right), 0\right\} \text {. }
$$

\section{Average Secrecy Capacity}

The ASC is derived as

$$
\bar{C}=\int_{0}^{\infty} \int_{0}^{\infty} C\left(\gamma_{S R A D}, \gamma_{S R A E}\right) f\left(\gamma_{S R A D}, \gamma_{S R A E}\right) d \gamma_{S R A D} d \gamma_{S R A E}=A_{1}+A_{2}-A_{3} .
$$

$A_{1}$ is given as

$$
\begin{aligned}
& A_{1}=\int_{0}^{\infty} \frac{1}{\gamma_{S R A D}} G_{2,2}^{1,2}\left(\gamma_{S R A D} \mid 1,0_{1,1}\right) G_{0,4}^{4,0}\left[\frac{\gamma_{S R A D}}{\chi_{D}} \mid 1,1,1,1_{-}\right] G_{1,5}^{4,1}\left[\frac{\gamma_{S R A D}}{\chi_{E}} \mid 1,1,1,1,0_{1}\right] d \gamma_{S R A D} \\
& =G_{2,2: 0,4: 1,5}^{2,1: 4,0: 4,1}\left[\begin{array}{c|c|c|c}
0,1 & - & 1 & \frac{1}{\chi_{D}}, \frac{1}{\chi_{E}}
\end{array}\right] .
\end{aligned}
$$



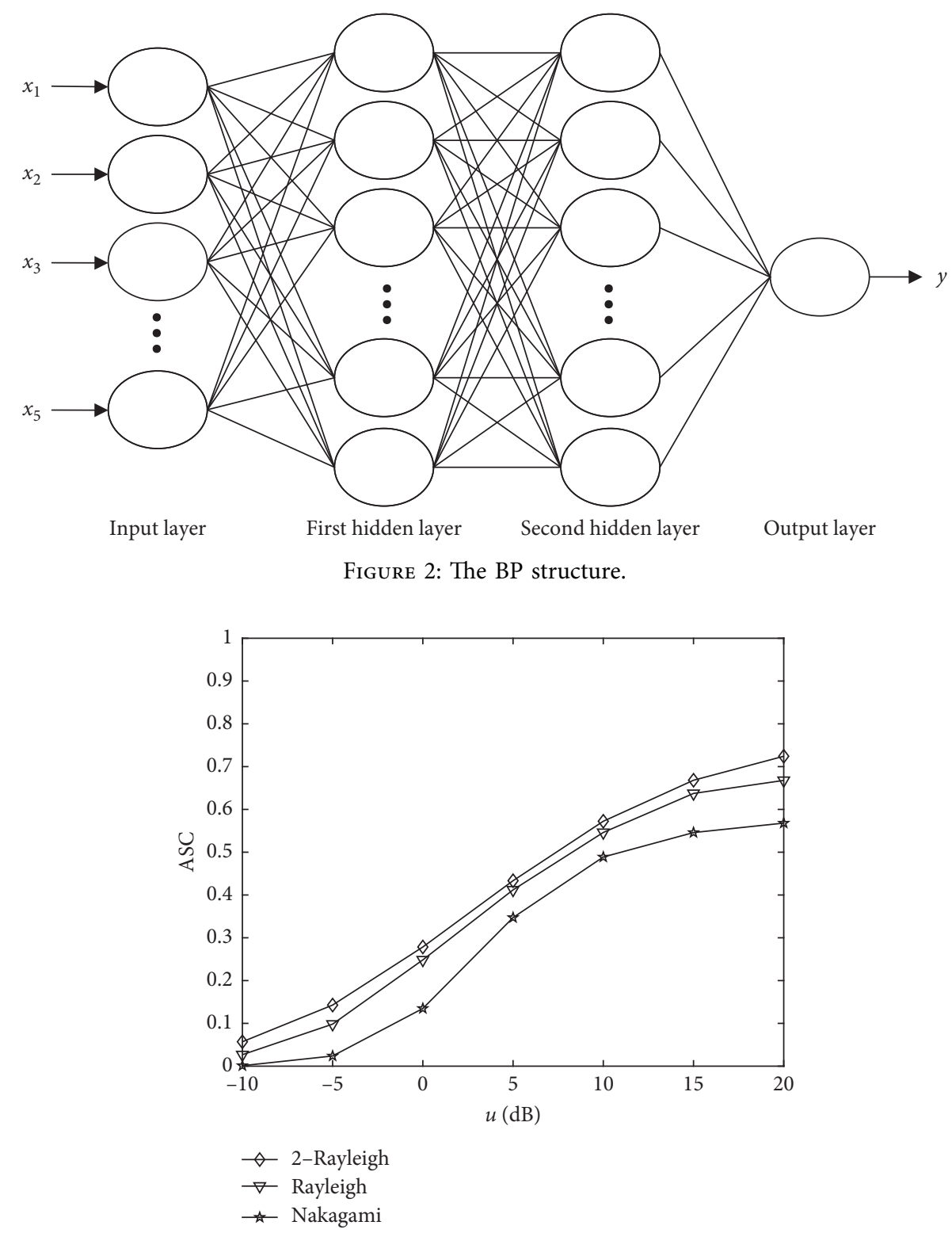

FIgURE 3: The ASC performance versus $(u)$.

We obtain the $A_{2}$ as

$$
\begin{aligned}
& A_{2}=\int_{0}^{\infty} G_{2,2}^{1,2}\left(\gamma_{\mathrm{SRAE}} \mid 1,0_{1,1}\right) \frac{1}{\gamma_{\mathrm{SRAE}}} G_{0,4}^{4,0}\left[\frac{\gamma_{\mathrm{SRAE}}}{\chi_{\mathrm{E}}} \mid 1,1,1,1_{-}\right] G_{1,5}^{4,1}\left[\frac{\gamma_{\mathrm{SRAE}}}{\chi_{\mathrm{D}}} \mid 1,1,1,1,0_{1}\right] d \gamma_{\mathrm{SRAE}} \\
& =G_{2,2: 0,4: 1,5}^{2,1: 4,0: 1,1}\left[\begin{array}{lll|l|l}
0,1 & - & 1 & \mid & \mid \frac{1}{\chi_{\mathrm{E}}}, \frac{1}{\chi_{\mathrm{D}}}
\end{array}\right] .
\end{aligned}
$$

We obtain the $A_{3}$ as

$$
A_{3}=\int_{0}^{\infty} \frac{1}{\gamma_{S R A E}} G_{2,2}^{1,2}\left(\gamma_{S R A E} \mid 1,0_{1,1}\right) G_{0,4}^{4,0}\left[\frac{\gamma_{S R A E}}{\chi_{E}} \mid 1,1,1,1_{-}\right] d \gamma_{S R A E}=G_{2,6}^{6,1}\left[\frac{1}{\chi_{E}} \mid 1,1,1,1,0,0_{0,1}\right] .
$$


TABLE 1: Simulation parameters.

\begin{tabular}{lc}
\hline Parameter & Value \\
\hline$m$ & $1,2,3$ \\
$K$ & 0.4 \\
$W_{\mathrm{SR}}$ & $5 \mathrm{~dB}$ \\
$W_{\mathrm{RE}}$ & $5 \mathrm{~dB}$ \\
\hline
\end{tabular}

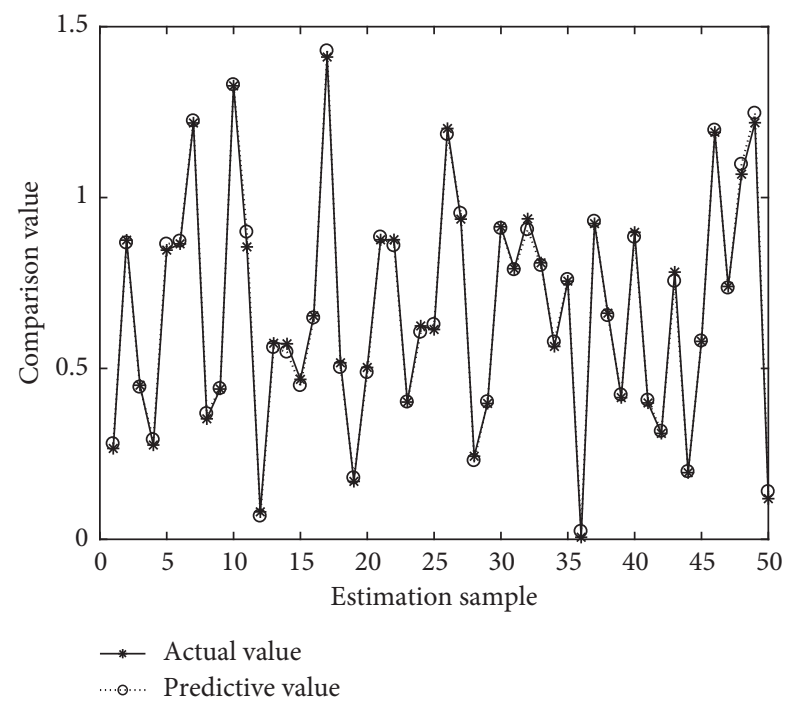

Figure 4: Prediction of BP.

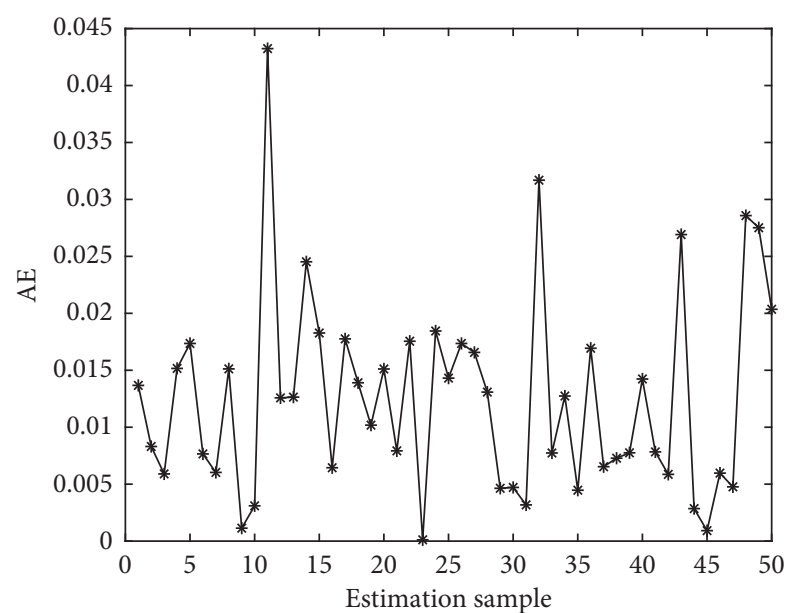

Figure 5: AE of BP.

Next, we use the derived ASC expressions to set up the data sets and design the BP prediction model.

\section{Secrecy Performance Prediction Method}

4.1. Data Sets. $T_{i}=\left(X_{i}, y_{i}\right) . X_{i}$ is given as

$$
X_{i}=\left(x_{i 1}, x_{i 2}, \ldots, x_{i 5}\right) \text {. }
$$

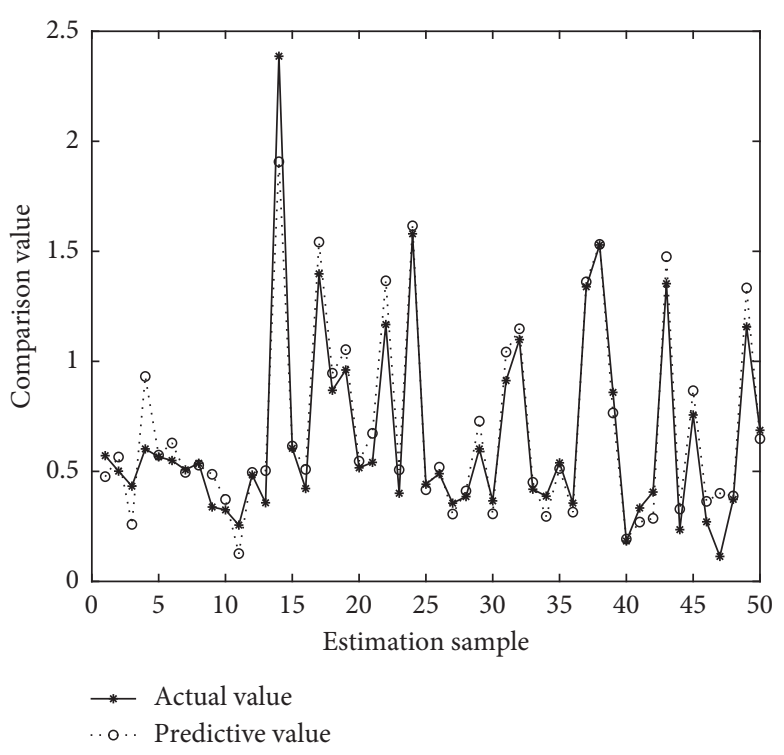

Figure 6: Prediction of ELM.

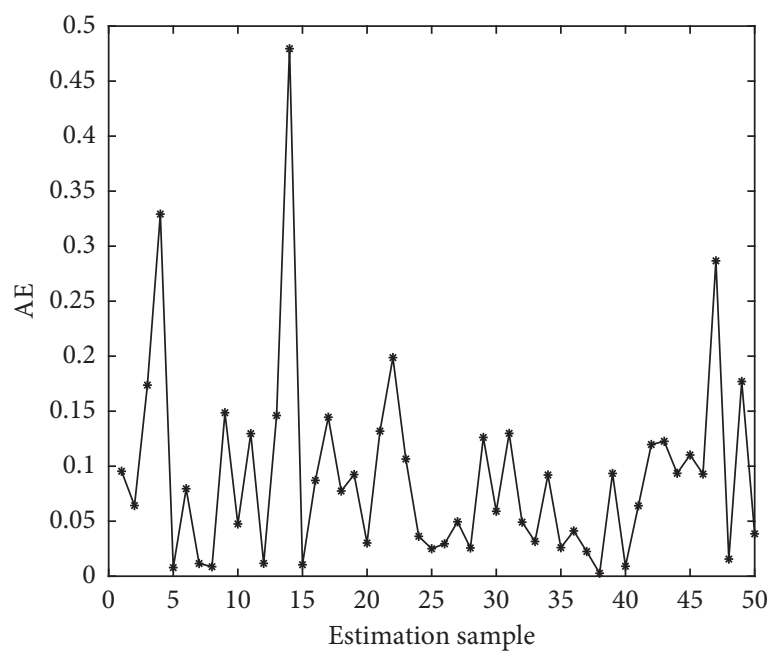

Figure 7: AE of ELM.

$X_{i}$ includes 5 indicators, which are $W_{\mathrm{SR}}, W_{\mathrm{RD}}, W_{\mathrm{RE}}, K$, and $\bar{\gamma}$. The ASC performance is the output $y_{i}$. By using (13), it can obtain the corresponding $y_{i}$.

4.2. Network Structure. Figure 2 shows the BP structure [24].

4.3. Metrics. Two metrics are MSE and AE. For PP testing data, they are given as

$$
\text { MSE }=\frac{\sum_{z=1}^{P P}\left(d^{z}-y^{z}\right)^{2}}{P P},
$$

$$
\mathrm{AE}=\left|d^{z}-y^{z}\right| .
$$




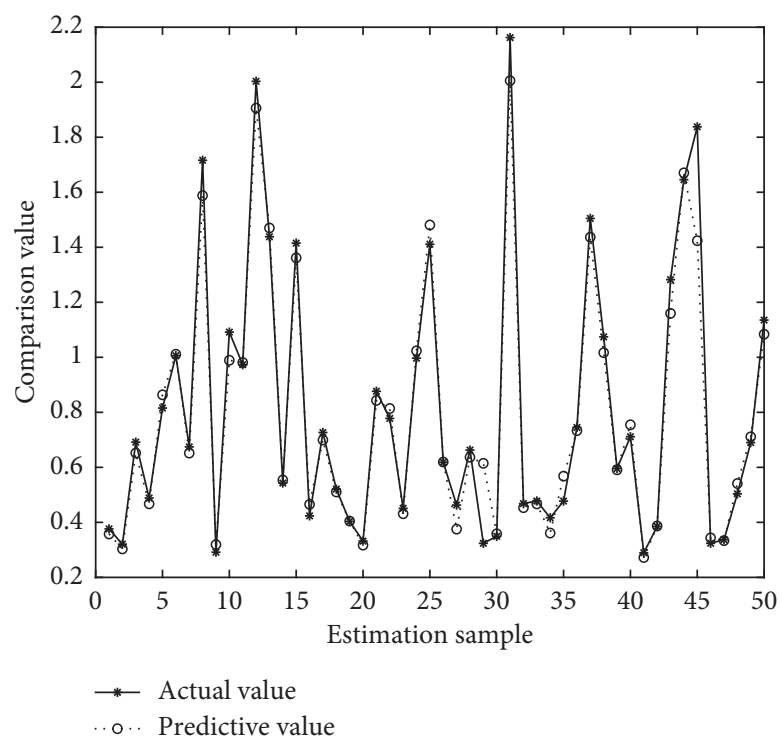

Figure 8: Prediction of SVM.

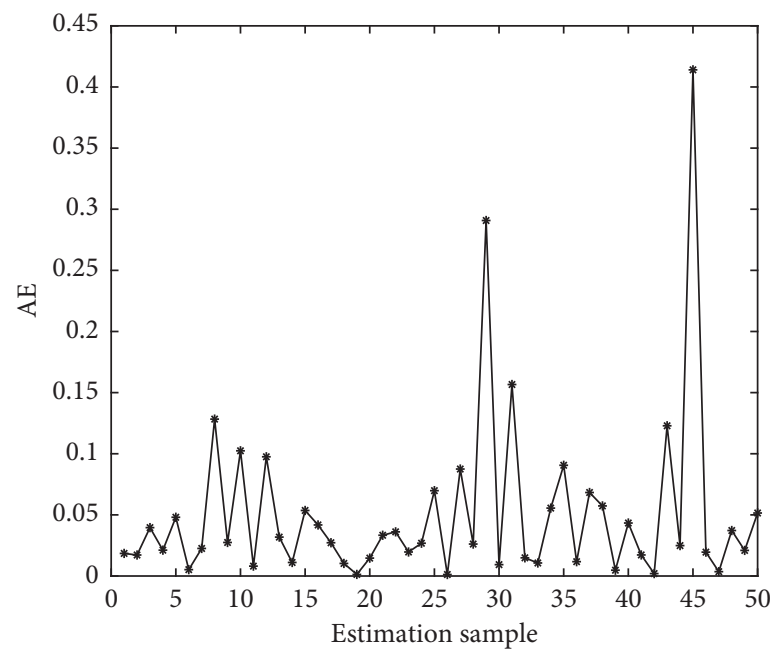

FIgure 9: AE of SVM.

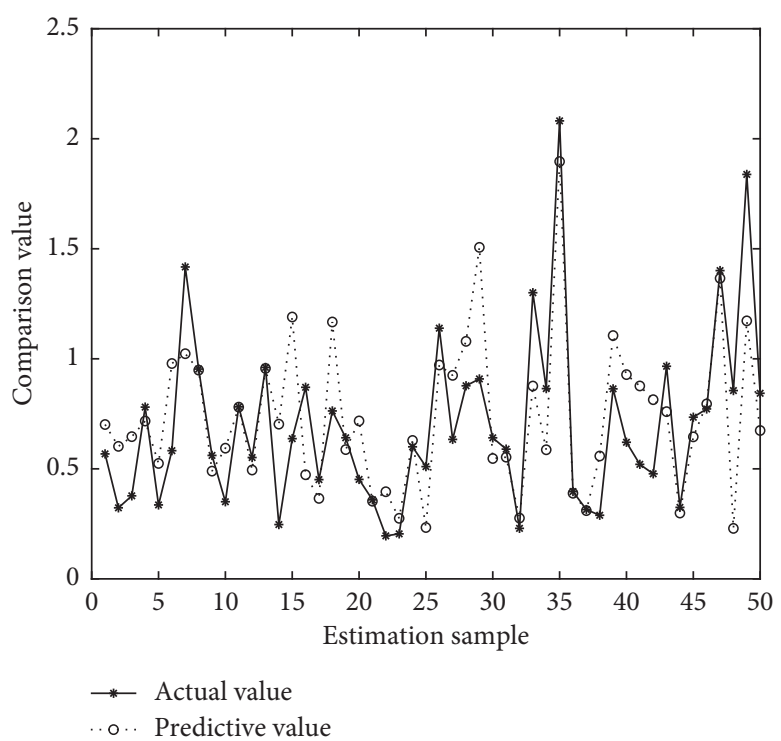

FIgURE 10: Prediction of GR. 


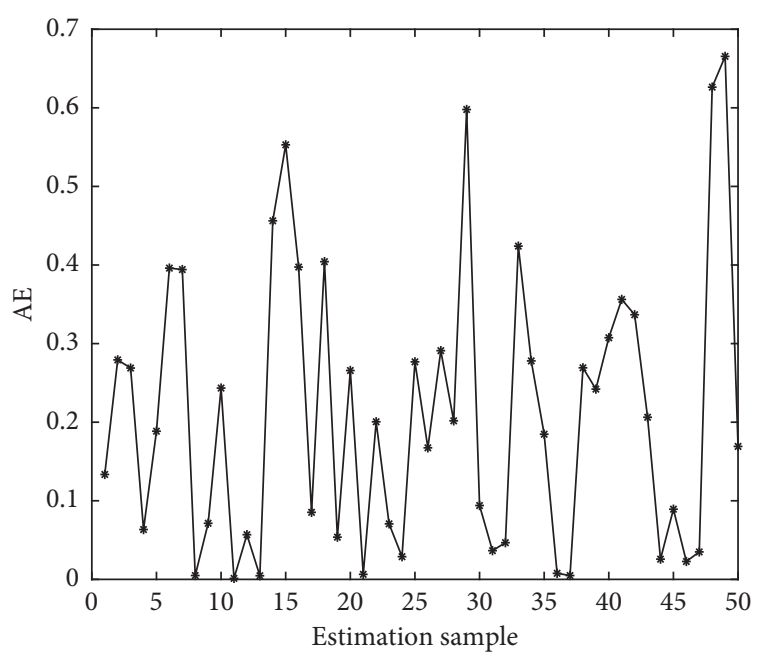

FIGURE 11: AE of GR.

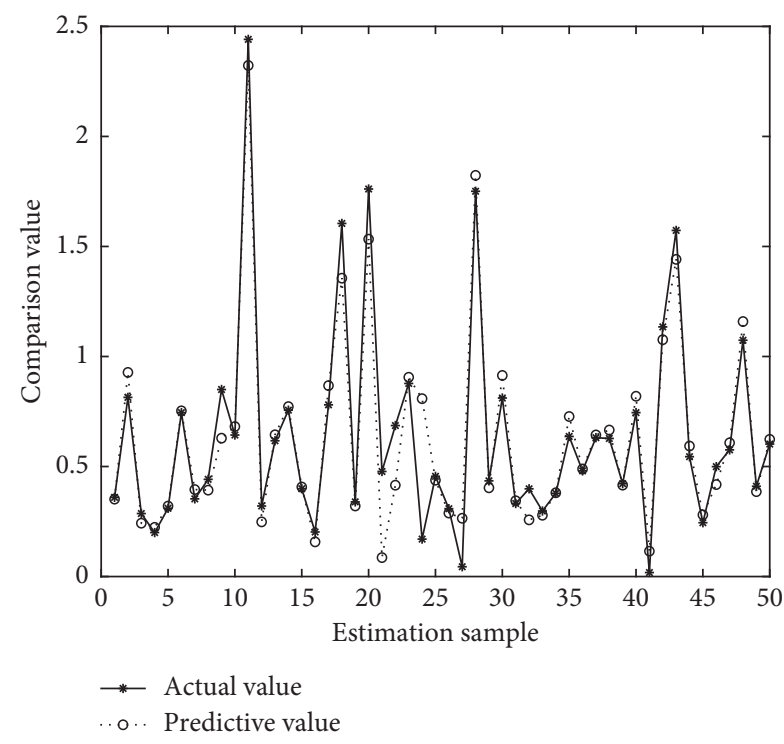

FIGURE 12: Prediction of RBF.

\section{Simulation Analysis}

Here, $E=1$, and $\mu=W_{\mathrm{RD}} / W_{\mathrm{RE}}$ (in decibels).

For different channels, we evaluate the ASC performance with $\bar{\gamma}=10 \mathrm{~dB}$ in Figure 3. The parameters are given in Table 1 . The following observations can be made: (1) increasing $\mu$ improves the ASC performance; (2) the 2-Rayleigh model can obtain the best ASC performance among the three channels.

In Figures 4-13, we consider SVM, ELM, GR, and RBF [25] methods to compare with the BP network. Table 2 gives the simulation parameters. The MSE and $\mathrm{AE}$ of $\mathrm{BP}$ are 0.000232889 and 0.04324 , which are the lowest MSE and AE in the five methods. This is because BP has the ability to adapt to the time-varying characteristics and enhance the global stability. It has more computing power than other four methods and can be used to solve the rapid optimization problem.

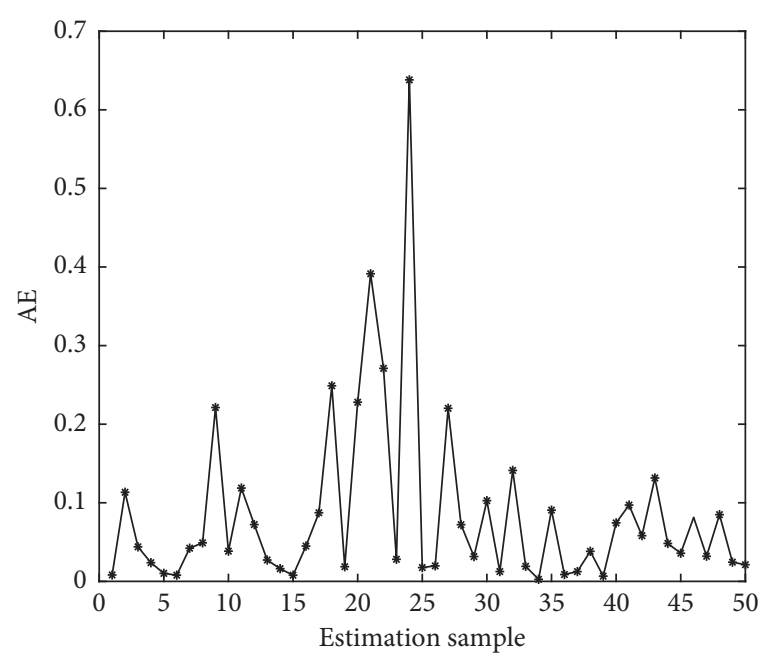

Figure 13: AE of RBF.

TABLE 2: The parameters for five different methods.

\begin{tabular}{|c|c|c|c|c|c|}
\hline Algorithm & $\mathrm{BP}$ & ELM & SVM & GR & $\mathrm{RBF}$ \\
\hline Parameter 1 & $\begin{array}{l}\mathrm{q}: 10 \\
\mathrm{r}: 10\end{array}$ & q: 18000 & c: 128 & Spread: 0.054 & Spread: 18 \\
\hline $\begin{array}{l}\text { Parameter } 2 \\
\text { Parameter } 3\end{array}$ & $\begin{array}{l}a_{1}: 0.01 \\
a_{2}: 0.01\end{array}$ & & g: 0.011 & & \\
\hline
\end{tabular}

\section{Conclusion}

An AF relaying scheme was used to improve the ASC performance of medical IoT communication networks in this paper. The ASC expressions were derived. Furthermore, we proposed an intelligent prediction algorithm based on the BP network. The simulation results show that (1) as the $u$ increases, the system's ASC performance becomes better and (2) compared with SVM, ELM, GR, and RBF methods, the proposed $\mathrm{BP}$ algorithm can obtain a better MSE and AE.

\section{Data Availability}

The data used to support the findings of this study are available from the corresponding author upon reasonable request and with permission of funders.

\section{Conflicts of Interest}

The authors declare that they have no conflicts of interest.

\section{Acknowledgments}

This work was supported by the National Natural Science Foundation of China (no. 11664043).

\section{References}

[1] R. Cao, Z. Tang, C. Liu, and B. Veeravalli, "A scalable multicloud storage architecture for cloud-supported medical Internet of Things," IEEE Internet of Things Journal, vol. 7, no. 3, pp. 1641-1654, 2020.

[2] H. Wang, L. Xu, Z. Yan, and T. A. Gulliver, "Low-complexity MIMO-FBMC sparse channel parameter estimation for 
industrial big data communications," IEEE Transactions on Industrial Informatics, vol. 17, no. 5, pp. 3422-3430, 2021.

[3] D. Meng, X. Wang, M. Huang, L. Wan, and B. Zhang, "Robust weighted subspace fitting for DOA estimation via block sparse recovery," IEEE Communications Letters, vol. 24, no. 3, pp. 563-567, 2020.

[4] X. Wang, L. Wan, M. Huang, C. Shen, Z. Han, and T. Zhu, "Low-complexity channel estimation for circular and noncircular signals in virtual MIMO vehicle communication systems," IEEE Transactions on Vehicular Technology, vol. 69, no. 4, pp. 3916-3928, 2020.

[5] X. Wang, M. Huang, and L. Wan, "Joint 2D-DOD and 2DDOA estimation for coprime EMVS-MIMO radar," Circuits, Systems and Signal Processing, vol. 40, no. 6, pp. 2950-2966, 2021.

[6] C. W. Chen, "Internet of video Things: next-generation IoT with visual sensors," IEEE Internet of Things Journal, vol. 7, no. 8, pp. 6676-6685, 2020.

[7] P. Gope, Y. Gheraibia, S. Kabir, and B. Sikdar, "A secure IoTbased modern healthcare system with fault-tolerant decision making process," IEEE Journal of Biomedical and Health Informatics, vol. 25, no. 3, pp. 862-873, 2021.

[8] H.-M. Wang, Q. Yang, Z. Ding, and H. V. Poor, "Secure shortpacket communications for mission-critical IoT applications," IEEE Transactions on Wireless Communications, vol. 18, no. 5, pp. 2565-2578, 2019.

[9] M. Torabi and D. Haccoun, "Impact of antenna correlation on the physical layer security of cooperative relaying with OSTBC system," IET Communications, vol. 14, no. 19, pp. 3472-3479, 2020.

[10] S. Park and H. Son, "Near-Perfect code scrambling with limited key information for wiretap channels," IEEE Transactions on Vehicular Technology, vol. 69, no. 11, pp. 1341013423, 2020.

[11] W. Yang, X. Zhao, and J. He, "Physical layer security and energy efficiency driven resource optimisation for cognitive relay networks," IET Communications, vol. 14, no. 17, pp. 2953-2961, 2020.

[12] C. Zhang, F. Jia, Z. Zhang, J. Ge, and F. Gong, "Physical layer security designs for $5 \mathrm{G}$ NOMA systems with a stronger nearend internal eavesdropper," IEEE Transactions on Vehicular Technology, vol. 69, no. 11, pp. 13005-13017, 2020.

[13] X. Lu, W. Yang, X. Guan, and Y. Cai, "DCE-based secure transmission for massive MIMO relay system Against active eavesdropper," IEEE Transactions on Vehicular Technology, vol. 69, no. 11, pp. 13045-13059, 2020.

[14] L. Xu, X. Yu, and T. A. Gulliver, "Intelligent outage probability prediction for mobile IoT networks based on an IGWOelman neural network," IEEE Transactions on Vehicular Technology, vol. 70, no. 2, pp. 1365-1375, 2021.

[15] L. Xu, H. Wang, and T. A. Gulliver, "Outage probability performance analysis and prediction for mobile IoV networks based on ICS-BP neural network," IEEE Internet of Things Journal, vol. 8, no. 5, pp. 3524-3533, 2021.

[16] X. J. Peng, "A spheres-based support vector machine for pattern classification," Neural Computing \& Applications, vol. 31, no. 1, pp. 379-396, 2019.

[17] T. Wu, W. L. Xue, H. Z. Wang et al., "Extreme learning machine-based state reconstruction for automatic attack filtering in cyber physical power system," IEEE Transactions on Industrial Informatics, vol. 17, no. 3, pp. 1892-1904, 2021.

[18] L. Xu, H. Wang, H. Li, W. Lin, and T. A. Gulliver, "QoS intelligent prediction for mobile video networks: a GR approach," Neural Computing and Applications, vol. 33, no. 9, pp. 3891-3900, 2021.

[19] G. K. Karagiannidis, N. C. Sagias, and P. T. Mathiopoulos, "Nakagami: a novel stochastic model for cascaded fading channels," IEEE Transactions on Communications, vol. 55, no. 8, pp. 1453-1458, 2007.

[20] A. Pandey and S. Yadav, "Physical layer security in cooperative AF relaying networks with direct links over mixed Rayleigh and double-Rayleigh fading channels," IEEE Transactions on Vehicular Technology, vol. 67, no. 11, pp. 10615-10630, 2018.

[21] F. K. Gong, Y. Wang, N. Zhang, and P. Ye, "Cooperative mobile-to-mobile communications over double Nakagami-m fading channels," IET Communications, vol. 6, no. 18, pp. 3165-3175, 2012.

[22] I. S. Gradshteyn and I. M. Ryzhik, Table of Integrals, Series and Products, Academic, San Diego, CA, USA, 2007.

[23] M. Bloch, J. Barros, M. R. D. Rodrigues, and S. W. McLaughlin, "Wireless information-theoretic security," IEEE Transactions on Information Theory, vol. 54, no. 6, pp. 2515-2534, 2008.

[24] X. Liu, Y. Zhou, Z. Wang, and X. Chen, "A BP neural network-based communication blind signal detection method with cyber-physical-social systems," IEEE Access, vol. 6, pp. 43920-43935, 2018.

[25] X. Zeng, H. Peng, and F. Zhou, "A regularized SNPOM for stable parameter estimation of RBF-AR(X) model," IEEE Transactions on Neural Networks and Learning Systems, vol. 29, no. 4, pp. 779-791, 2018. 